\title{
Non-Convex Economic Dispatch with Prohibited Operating Zones through Gravitational Search Algorithm
}

\author{
P.K. Hota*, N.C. Sahu** \\ * Department of Electrical Engineering, Veer Surendra Sai University of Technology, Burla, India \\ ** Department of Electrical Engineering, ITER, SOA University, Bhubaneswar, India
}

\begin{tabular}{l}
\hline Article Info \\
\hline Article history: \\
Received Dec 28, 2014 \\
Revised Jun 29, 2015 \\
Accepted Jul 18, 2015 \\
\hline
\end{tabular}

\section{Keyword:}

Economic load dispatch Evolutionary programming Gravitational search algorithm Prohibited operating zones Valve point effects

\begin{abstract}
This paper presents a new approach to the solution of optimal power generation for economic load dispatch (ELD) using gravitational search algorithm (GSA) when all the generators include valve point effects and some/all of the generators have prohibited operating zones. In this paper a gravitational search algorithm is suggested that deals with equality and inequality constraints in ELD problems. A constraint treatment mechanism is also discussed to accelerate the optimization process. To verify the robustness and superiority of the proposed GSA based approach, a practical sized 40-generators case with valve point effects and prohibited operating zones is considered. The simulation results reveal that the proposed GSA approach ensures convergence within an acceptable execution time and provides highly optimal solution as compared to the results obtained from well established heuristic optimization approaches.
\end{abstract}

Copyright $(2015$ Institute of Advanced Engineering and Science. All rights reserved.

\section{Corresponding Author:}

P.K.Hota,

Department of Electrical Engineering,

Veer Surendra Sai University of Technology,

Burla, PIN: 768018, Odisha, India.

Email: p_hota@rediffmail.com

\section{INTRODUCTION}

Economic load dispatch is an important power system optimization task and one of the fundamental issues of power system operation for scheduling generation among the committed generators while satisfying system constraints and minimizing the cost of energy requirements. For solving ELD problems, previously classical methods [1] have been successfully employed with some approximations due to nonlinear characteristics of practical systems [2]. However, such approximations may cause to huge revenue loss over the passage of time. The classical mathematical programming such as linear programming, quadratic programming and interior point algorithm, etc., produce promising economic generation scheduling results when the fuel cost curve is considered as monotonically increasing one. However, when the problem is highly nonlinear or has non-smooth cost functions, some of these techniques may not be able to produce good solutions.

In past two decades, stochastic search algorithms like genetic algorithm (GA) [3], evolutionary programming (EP) [4] and simulated annealing [5] may prove to be very efficient in solving complex ELD problems but its control parameters tuning is difficult task. Tabu search [6], particle swarm optimization [7][8] and neural network approaches [9]-[11] have been applied successfully but, these methods do not always guarantee to have the globally optimal solution. The recent research has identified few drawbacks of the stochastic methods like premature convergence of GA causing degradation in performance and reduction its search capability and unsuitable when applied to highly epistatic objective functions (i.e., where the parameters being optimized are highly correlated). Many researchers have solved the ELD problem with valve point effects of generators efficiently by using the above mentioned heuristic optimization techniques. 
But, in all these methods, the whole of the unit operating range is available for operation. In practice, the operating range is broken into several disjoint sub-regions when prohibited zones are present. The fuel cost curve of a unit with prohibited operating zones is a discontinuous function. Thus, the traditional methods cannot be directly employed to solve this dispatch problem. However, the heuristic search techniques such as GA, SA, PSO, etc., are capable of taking into account the unit's prohibited zones, since they do not require the function to be continuous.

Orero, et al. [12] have applied genetic algorithm approach to solve the economic dispatch of generators with prohibited operating zones. In this paper, they have used the penalty function approach to handle the prohibited operating zone constraint. Chen, et al. [13] have also solved the same problem using genetic algorithm where, ramp-rate limits are also considered apart from prohibited operating zones. Evolutionary programming based economic dispatch of generator with prohibited operating zones has been proposed by Jayabarathi, et al. [14]. In another paper, Pereira-Neto, et al. [15] have used an efficient evolutionary strategy optimization procedure to solve the non-convex ELD problem with prohibited operating zone constraint. In the above mentioned techniques, only small size ELD problems with prohibited operating zones have been solved. However, Chaturvedi, et al. [16] have solved a large scale non-convex ELD problem with prohibited operating zones using a self-organizing hierarchical PSO technique. Similarly, Selvakumar, et al. [17] have proposed a new particle swarm optimization (NPSO) solution procedure to nonconvex ELD problem with prohibited operating zone constraint. Coelho, et al. [18] have combined chaotic differential evolution and quadratic programming technique for economic dispatch optimization with valve point effect. Recently, a new heuristic search algorithm, namely gravitational search algorithm (GSA) motivated by gravitational law and law of motion has been proposed by Rashedi, et al. [19]. They have been applied successfully in solving various non linear functions. Recently, GSA has been successfully applied to ELD and hydrothermal scheduling problems [20]-[22]. The obtained results confirm the high performance and efficient convergent characteristics of the proposed method. Further, GSA has a flexible and wellbalanced mechanism to enhance exploration ability. Main objective of this paper is to present the use of GSA optimization technique in obtaining the ELD results.

Hence, an attempt has been made in this paper to explore the possibility of applying recent heuristic optimization technique namely gravitational search algorithm in solving the large scale non-convex ELD problem with prohibited operating zones. A 40-unit non-convex ELD problem with all practical constraints such as ramp-rate constraint, prohibited operating zone constraint, etc., has been solved effectively using gravitation search algorithm in this paper. To investigate the potential of the proposed approach, the simulation results are compared to that of recent approaches reported in the literature. The proposed methodology gives the cheapest generation schedule and outperforms previously reported other methods particularly when applied to large-scale ELD problems.

\section{PROBLEM FORMULATION}

ELD problem is about minimizing the fuel cost of generating unit real power outputs for a specified period of operation so as to accomplish optimal dispatch among the committed units, while satisfying the system constraints. The generators with multiple valve steam turbines possess a wide variation in the inputoutput characteristics. The valve point effect introduces ripples in the heat rate curves and cannot be represented by the polynomial function. Hence, the actual cost curve is a combination of sinusoidal function and quadratic function represented by the following equation.

$$
f_{i}\left(P_{i}\right)=a_{i}+b_{i} P_{i}+c_{i} P_{i}^{2}+\left|e_{i} \times \sin \left(f_{i} \times\left(P_{i, \min }-P_{i}\right)\right)\right|
$$

Where, $a_{i}, b_{i}$ and $c_{i}$ are the fuel-cost coefficients of the $i^{\text {th }}$ unit and $e_{i}, f_{i}$ are the constants of the $i^{\text {th }}$ unit with valve point effects.

The prime objective of the ELD problem is to determine the most economic loadings of generators to minimize the generation cost such that the load demands $P_{D}$ in the scheduling horizon can be met and simultaneously the operation constraints are satisfied. Here, this constrained optimization problem can be written as:

$$
\text { Minimize } F=\sum_{i \in \Omega} f_{i}\left(P_{i}\right)
$$

Where, $\Omega$ is the set of all committed units. This minimization problem is subjected to a variety of constraints depending upon assumptions and practical implications like power balance constraints, generator output limits, ramp rate limits and prohibited operating zones. These constraints and limits are discussed as follows: 
1) Power balance constraint or demand constraint: The total generation should be equal to the total system demand $P_{D}$ plus the transmission loss $P_{\text {LOSS. }}$. That is represented as:

$$
\sum_{i \in \Omega} P_{i}=P_{D}+P_{L O S S}
$$

The transmission loss must be taken into account in order to achieve true economic dispatch. To calculate the transmission loss, $B$ coefficients method is used in general. The loss is represented by $B$ coefficients.

$$
P_{\text {LOSS }}=\sum_{i \in \Omega} \sum_{j \in \Omega} P_{i} B_{i j} P_{j}+\sum_{i \in \Omega} B_{0 i} P_{i}+B_{00}
$$

2) The generator limits: The generation output of each unit should be between its minimum and maximum limits. That is, the following inequality constraint for each generator should be satisfied.

$$
P_{i, \min } \leq P_{i} \leq P_{i, \max } \quad \forall i \in(\Omega-\theta)
$$

Where, $\theta$ is the set of all committed units having prohibited zones, $(\Omega-\theta)$ is the set of all committed units which are not having prohibited zones, $P_{i}$ is the power output of $i^{\text {th }}$ generator and $P_{i, \min }$ and $P_{i, \max }$ are the minimum and maximum real power output of $i^{\text {th }}$ generator.

3) Ramp rate limits: In ELD problems, the generator output is usually assumed to be adjusted smoothly and instantaneously. However, under practical circumstances ramp rate limit restricts the operating range of all the online units for adjusting the generation operation between two operating periods. In other words, this constraint restricts the operating range of the physical lower and upper limit to the effective lower limit $P_{\text {effmin }, i}$ and upper limit $P_{\text {effmax }, i}$, respectively.

These limits [15] are defined as:

$$
\begin{aligned}
& P_{\text {effmin }, i}=\max \left[P_{i, \min }, P_{i 0}-D R_{i}\right] \\
& P_{\text {effmax }, i}=\min \left[P_{i, \max }, P_{i 0}+U R_{i}\right]
\end{aligned}
$$

Where, $P_{i}$ and $P_{i 0}$ are the current and previous power output of $i^{\text {th }}$ generator, respectively; $D R_{i}$ and $U R_{i}$ are the down ramp and up ramp limits of the $i^{\text {th }}$ generator as generation decreases and increases, respectively. Accordingly, it is obtained as:

$$
P_{\text {effmin }, i} \leq P_{i} \leq P_{\text {effmax }, i}
$$

4) Prohibited operating zones: The input-output characteristics of modern units are inherently nonlinear because of the steam valve point loadings. The operating zones due to valve point loading or vibration due to shaft bearing is generally avoided in order to achieve best economy, called prohibited operating zones of a unit, which make the cost curve discontinuous in nature. The prohibited operating zone constraints are described as:

For all $i \in \theta$

$$
\begin{aligned}
& P_{i, \min } \leq P_{i} \leq P_{i, 1}^{l} \\
& P_{i, k-1}^{u} \leq P_{i} \leq P_{i, k}^{l} \quad k=2, \ldots, n_{i} \\
& P_{i, n_{i}}^{u} \leq P_{i} \leq P_{i, \max }
\end{aligned}
$$

Where, $P_{i, k}^{l}$ and $P_{i, k}^{u}$ are the lower and upper limits of $k^{\text {th }}$ prohibited zone for $i^{\text {th }}$ unit and $n_{i}$ is the number of prohibited zones of unit $i$.

The prohibited operating zone constraints (9-11) avoid the operation of units in the prohibited zones. The prohibited zones $n_{i}$ of the dispatchable units divide the operating region between the minimum and maximum generation limits into $n_{i}+1$ disjoint operating sub-regions. The proposed method for minimizing Equation (2) with constraints defined by Equation (3), (5-11) is presented in the following section. 


\section{GSA BASED ECONOMIC DISPATCH}

In this section, a brief description of GSA, the procedure for GSA based economic load dispatch, constraint satisfaction technique and the overall computational procedure have been explained.

\subsection{Gravitational Search Algorithm}

The gravitational search algorithm (GSA), is one of the recent heuristic search algorithm developed by Rashedi et al. in 2009 [17]. GSA is based on the physical law of gravity and the law of motion. The gravitational force between two particles is directly proportional to the product of their masses and inversely proportional to the square of the distance between them. In the proposed algorithm, agents are considered as objects and their performance is measured by their masses:

$$
P_{i}=\left(p_{i}, \cdots p_{i}^{d}, \cdots, p_{i}^{n}\right), i=1,2, \cdots m
$$

Where, $P_{i}^{d}$ is the position of the $i^{\text {th }}$ mass in the $d^{\text {th }}$ dimension and $n$ is the dimension of the search space. At specific time ' $t$ ' a gravitational force form mass $j$ act on mass $i$ and is defined as follows:

$$
F_{i j}^{d}(t)=G(t) \frac{M_{p i}(t) \times M_{a j}(t)}{R_{i j}(t)+\varepsilon}\left(p_{j}^{d}(t)-p_{i}^{d}(t)\right)
$$

Where, $M_{p i}$ is the passive gravitational mass related to agent $i, M_{a j}$ is the active gravitational mass related to agent $j, G(\mathrm{t})$ is the gravitational constant at time $t, R_{i j}(t)$ is the Euclidian distance between the two objects $i$ and $j$, and $\varepsilon$ is a small constant.

$$
R_{i j}(t)=\left\|P_{i}(t), P_{j}(t)\right\|_{2}
$$

The total force acting on the agent $i$ in the dimension $d$ is calculated as follows.

$$
F_{i}^{d}(t)=\sum_{j=1, j \neq i}^{m} \operatorname{rand}_{j} F_{i j}^{d}(t)
$$

Where, rand $_{j}$ is a random number in the interval $[0,1]$. According to the law of motion, the acceleration of the agent $i$, at time $t$, in the $d^{\text {th }}$ dimension, $\alpha_{i}^{d}(t)$ is given as follows:

$$
\alpha_{i}^{d}(t)=\frac{F_{i}^{d}(t)}{M_{i j}(t)}
$$

The next velocity of an agent is a function of its current velocity added to its current acceleration. Therefore, the next position and next velocity of an agent can be calculated as follows:

$$
\begin{aligned}
& v_{i}^{d}(t+1)=\operatorname{rand}_{i} p v_{i}^{d}(t)+\alpha_{i}^{d}(t) \\
& p_{i}^{d}(t+1)=p_{i}^{d}(t)+v_{i}^{d}(t+1)
\end{aligned}
$$

Where, rand $_{i}$ is a uniform random variable in the interval $[0,1]$. The gravitational constant, $G$, is initialized at the beginning and will be decreased with the time to control the search accuracy. In other words, $G$ is function of the initial value $\left(G_{0}\right)$ and time $(t)$ :

$$
\begin{aligned}
& G(t)=G\left(G_{0}, t\right) \\
& G(t)=G_{0} e^{-\alpha t / T}
\end{aligned}
$$

The masses of the agents are calculated using fitness evaluation. A heavier mass means a more efficient agent. This means that better agents have higher attractions and moves more slowly. Supposing the equality of the gravitational and inertia mass, the values of masses is calculated using the map of fitness. The gravitational and inertial masses are updated by the following equations.

$$
m_{i}(t)=\frac{\operatorname{fit}_{i}(t)-\operatorname{worst}(t)}{\operatorname{best}(t)-\operatorname{worst}(t)}
$$




$$
M_{i}(t)=\frac{m_{i}(t)}{\sum_{j=1}^{m} m_{j}}(t)
$$

Where, $f_{i t}(t)$ represents the fitness value of the agent $i$ at time $t$, and the $\operatorname{best}(t)$ and $\operatorname{worst}(t)$ in the population respectively, indicate the strongest and the weakness agent according to their fitness value. For a minimization problem:

$$
\begin{aligned}
& \operatorname{best}(t)=\min _{j \in\{1, \ldots, m\}} \text { fit }_{j}(t) \\
& \operatorname{worst}(t)=\max _{j \in\{1, \ldots, m\}} \text { fit }_{j}(t)
\end{aligned}
$$

\subsection{Gravitational Search Algorithm Based Economic Load Dispatch}

In order to handle the constraints conveniently, the structure of solutions for ELD problem solved by the proposed method is composed of a set of real power output decision variables for each unit in all over the scheduling periods. The section provides the solution methodology to the above-mentioned economic load dispatch problems through gravitational search algorithm.

\subsubsection{Initialization}

The initial population is carefully generated as it decides for reaching the optimum solution. It is composed of $m$ masses. The elements of each mass are the $n$-dimensional positions of search space. The elements of a mass are randomly created permutation of power outputs of the generating units. Each element is uniformly distributed within its feasible range. The initialization must satisfy all constraints given in section-2 and accordingly is generated as described below. $P_{i}$ is having uniformly distributed generation level ranging over $\left[P_{i, \min }, P_{i, \max }\right]$ for units with whole of its operating range available for operation. But, for all units with prohibited operating zones, initially a random integer number $u_{r}$ between 1 and $n_{i}+1$ both inclusive is generated. This number is the operating sub-region of unit $i$, in which its generation level should fall. Generation $P_{i}$ must satisfy constraint Eq. (9) if $u_{r}=1$. $P_{i}$ must satisfy constraint Equation (11) when $u_{r}=n_{i}+$ 1 whereas, all intermediate number generated by $u_{r}$ result in generation levels constrained by Eq. (10). The above mentioned initialization procedure leads to permutation of generation outputs confined to operating sub-regions alone. However, the initialized solutions, i.e., $n$-dimensional masses are $P_{i}=\left[P_{1}, P_{2}, \ldots, P_{n}\right], i=$ $1,2, \ldots, m$ and $n$-the number of generating units. In order to satisfy the exact power balance constraint (Eq. 3 ), usually the largest generator without prohibited operating zones is arbitrarily selected as a dependent unit. Accordingly, its output is calculated as:

$$
P_{d}=P_{D}+P_{\text {LOSS }}-\sum_{\substack{i=1 \\ i \neq d}}^{n} P_{i}
$$

The power loss $P_{\text {LOSS, }}$ is obtained using the $B$-matrix loss formula as described by Equation (4).

\subsubsection{Fitness Evaluation (Objective Function)}

The fitness evaluation in each agent in the population set is evaluated using the Equation (2). Iteration count from this step, $t=1$. Update $G(t)$, best $(t)$, worst $(t)$ and $M_{i}(t)$ for $i=1,2 \ldots m$

\subsubsection{Agent Force Calculation}

The total force acting on the agent $i$ in the dimension $d$ is calculated in Equation (15).

\subsubsection{Evaluation of Acceleration of an Agent}

The acceleration of an agent in $d^{\text {th }}$ dimension over $T$ dispatch period has evaluated using Equation

\subsubsection{Update the Agents' Position}

The next velocity of an agent is calculated by adding the acceleration of an agent to the current velocity and also position of an agent will updated.

\subsubsection{Stopping Criterion}

There are various criteria available to stop a stochastic optimization algorithm. In this paper, to compare with the previous results, maximum number of iterations is chosen as the stopping criterion. If the 
stopping criterion is not satisfied, the above procedure is repeated from fitness evaluation with incremented iteration.

\subsection{Constraints Satisfaction Technique}

The elements of initial masses contain generated powers only within the operating sub-regions. However, after updating process of GSA algorithm, they may violate the constraints given by Equations, (511). The procedure for constraints satisfaction is dealt with as follows [12].

If generator limits constraint (5) is violated then,

$$
P_{i}=P_{i, \min } \text { if } P_{i}<P_{i, \min } \text { and } P_{i}=P_{i, \max } \text { if } P_{i}>P_{i, \max }
$$

If prohibited operating zones constraint (9-11) are violated, then the mid-points of the prohibited operating zones for each generator are computed. The mid-points of the prohibited zone corresponding to a generation level $P_{i}$ lying between $P_{i, n}^{l}$ and $P_{i, n}^{u}$ is given as:

$$
\begin{aligned}
& M_{i, n}=\frac{P_{i, n}^{u}+P_{i, n}^{l}}{2} \text { for } n=1,2, \ldots, n_{i} \text { and } \\
& P_{i}=P_{i, n}^{l} \text { if } P_{i}<M_{i, n} \text { and } P_{i}=P_{i, n}^{u} \text { if } P_{i}>M_{i, n}
\end{aligned}
$$

If ramp-rate limits constraints are violated, then the limits $P_{i, \min }, P_{i, \max }$ in Equation (26) are replaced by $P_{\text {effmin, } i}$ and $P_{e f f m a x, i}$ to satisfy these constraints.

\subsection{Computational Procedure}

The purposed GSA approach for economic load dispatch problem with valve- point effect and prohibited operating zones can be summarized as follows.

Step 1. Search space identification

Step 2. Generate initial population between minimum and maximum values.

Step 3. Fitness evaluation of agents.

Step 4. Update gravitational constant $G(t)$, best $(t)$ and worst $(t)$ in the population and update the mass of the object $M_{i}(t)$.

Step 5. Force calculation in different direction.

Step 6. Calculation of acceleration and velocity of an agent.

Step 7. Updating the position of an agent.

Step 8. Repeat step 3 to step 7 until the stop criteria is satisfied

Step 9. Stop.

\section{SYSTEM AND RESULTS}

The present work has been implemented in command line in Matlab-7.0 for the solution of economic load dispatch with non-smooth cost functions. The program was run on a 3.06GHz, Pentium-IV, with 256MB RAM PC. After several trials, the setup for the proposed algorithm is executed with following parameters:

$m=100$ (masses), $G$ is set using Eq. (20), where $G_{0}$ is set to 100 and $\alpha$ is set to 8 , and $T$ is the total number of iterations with a maximum value of 1000 .

To demonstrate the robustness of the proposed approach, a practical sized test system consisting of 40 generators with valve point loading effects, ramp rate limits constraints and prohibited operating zones is considered. A load demand of 10,500 MW is considered in this case. The input data of 40 units is shown in Table 1 and 2. The optimal results by the proposed GSA algorithm are compared with those obtained from seven other methods [14]-[16] and shown in Table 3. The other well established heuristic methods are improved fast evolutionary programming (IFEP) [15], modified particle swarm optimization (MPSO) [15], particle swarm optimization-local random search (PSO-LRS) [15], new particle swarm optimization (NPSO) [15], new particle swarm optimization-local random search (NPSO-LRS) [15], self-organizing hierarchical particle swarm optimization (SOHPSO) [14] and chaotic differential evolution (DE) [16] The minimum cost obtained by proposed GSA is $121,447.547 \$ / \mathrm{h}$ whereas, the minimum costs obtained by these seven methods are more than the proposed GSA method. Therefore, the GSA method gives the cheapest generation schedule, which may be considered as global one. The generation outputs and corresponding cost of the optimal solution by proposed GSA method are provided in Table 4 . Hence, Table 3 and 4 validate the superiority of the GSA method. 


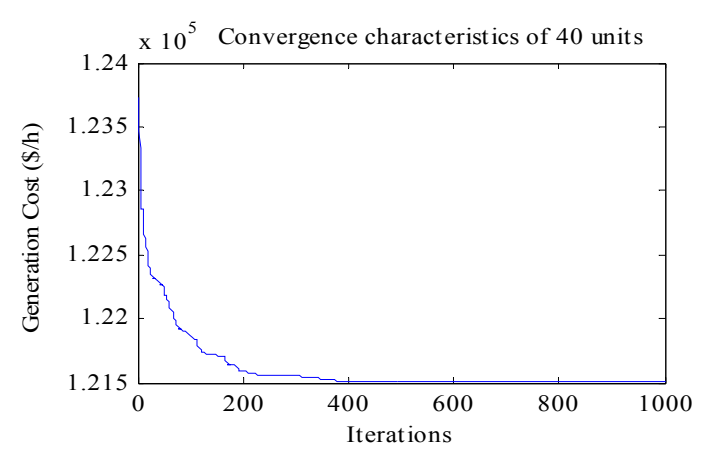

Figure 1. Convergence characteristics of the proposed GSA method

Table 1. 40-unit system with valve point loading effect

\begin{tabular}{|c|c|c|c|c|c|c|c|}
\hline Unit & $P i$, min & $P i, \max$ & $a$ & $b$ & $c$ & $e$ & $f$ \\
\hline 1 & 36 & 114 & 0.00690 & 6.73 & 94.705 & 100 & 0.084 \\
\hline 2 & 36 & 114 & 0.00690 & 6.73 & 94.705 & 100 & 0.084 \\
\hline 3 & 60 & 120 & 0.02028 & 7.07 & 309.54 & 100 & 0.084 \\
\hline 4 & 80 & 190 & 0.00942 & 8.18 & 369.03 & 150 & 0.063 \\
\hline 5 & 47 & 97 & 0.0114 & 5.35 & 148.89 & 120 & 0.077 \\
\hline 6 & 68 & 140 & 0.01142 & 8.05 & 222.33 & 100 & 0.084 \\
\hline 7 & 110 & 300 & 0.00357 & 8.05 & 287.71 & 200 & 0.042 \\
\hline 8 & 135 & 300 & 0.00492 & 6.99 & 391.98 & 200 & 0.042 \\
\hline 9 & 135 & 300 & 0.00573 & 6.60 & 455.76 & 200 & 0.042 \\
\hline 10 & 130 & 300 & 0.00605 & 12.9 & 722.82 & 200 & 0.042 \\
\hline 11 & 94 & 375 & 0.00515 & 12.9 & 635.20 & 200 & 0.042 \\
\hline 12 & 94 & 375 & 0.00569 & 12.8 & 654.69 & 200 & 0.042 \\
\hline 13 & 125 & 500 & 0.00421 & 12.5 & 913.40 & 300 & 0.035 \\
\hline 14 & 125 & 500 & 0.00752 & 8.84 & 1760.4 & 300 & 0.035 \\
\hline 15 & 125 & 500 & 0.00708 & 9.15 & 1728.3 & 300 & 0.035 \\
\hline 16 & 125 & 500 & 0.00708 & 9.15 & 1728.3 & 300 & 0.035 \\
\hline 17 & 220 & 500 & 0.00313 & 7.97 & 647.85 & 300 & 0.035 \\
\hline 18 & 220 & 500 & 0.00313 & 7.95 & 649.69 & 300 & 0.035 \\
\hline 19 & 242 & 550 & 0.00313 & 7.97 & 647.83 & 300 & 0.035 \\
\hline 20 & 242 & 550 & 0.00313 & 7.97 & 647.81 & 300 & 0.035 \\
\hline 21 & 254 & 550 & 0.00298 & 6.63 & 785.96 & 300 & 0.035 \\
\hline 22 & 254 & 550 & 0.00298 & 6.63 & 785.96 & 300 & 0.035 \\
\hline 23 & 254 & 550 & 0.00284 & 6.66 & 794.53 & 300 & 0.035 \\
\hline 24 & 254 & 550 & 0.00284 & 6.66 & 794.53 & 300 & 0.035 \\
\hline 25 & 254 & 550 & 0.00277 & 7.10 & 801.32 & 300 & 0.035 \\
\hline 26 & 254 & 550 & 0.00277 & 7.10 & 801.32 & 300 & 0.035 \\
\hline 27 & 10 & 150 & 0.52124 & 3.33 & 1055.1 & 120 & 0.077 \\
\hline 28 & 10 & 150 & 0.52124 & 3.33 & 1055.1 & 120 & 0.077 \\
\hline 29 & 10 & 150 & 0.52124 & 3.33 & 1055.1 & 120 & 0.077 \\
\hline 30 & 47 & 97 & 0.01140 & 5.35 & 148.89 & 120 & 0.077 \\
\hline 31 & 60 & 190 & 0.00160 & 6.43 & 222.92 & 150 & 0.063 \\
\hline 32 & 60 & 190 & 0.00160 & 6.43 & 222.92 & 150 & 0.063 \\
\hline 33 & 60 & 190 & 0.00160 & 6.43 & 222.92 & 150 & 0.063 \\
\hline 34 & 90 & 200 & 0.0001 & 8.95 & 107.87 & 200 & 0.042 \\
\hline 35 & 90 & 200 & 0.0001 & 8.62 & 116.58 & 200 & 0.042 \\
\hline 36 & 90 & 200 & 0.0001 & 8.62 & 116.58 & 200 & 0.042 \\
\hline 37 & 25 & 110 & 0.0161 & 5.88 & 307.45 & 80 & 0.098 \\
\hline 38 & 25 & 110 & 0.0161 & 5.88 & 307.45 & 80 & 0.098 \\
\hline 39 & 25 & 110 & 0.0161 & 5.88 & 307.45 & 80 & 0.098 \\
\hline 40 & 242 & 550 & 0.00313 & 7.97 & 647.83 & 300 & 0.035 \\
\hline
\end{tabular}

The convergence characteristic of proposed GSA method is illustrated in Figure 1. To assess the robustness and effectiveness of the proposed GSA method in comparison to other methods [14]-[16] in a statistical manner, the relative frequency of convergence is provided for each range of cost among 100 trials in Table 5. One can see the dominating nature of GSA method over other existing methods. The performance of GSA is compared with those of other heuristic methods. It is clear that the GSA method outperforms and provides the cheapest generation schedule for which huge revenue is saved over a long period, say yearly. The heuristic methods are stochastic methods where the solutions obtained may not be same at every run. When the program is run 100 times, the ranges of the cost of the system obtained are classified into 10 sub- 
ranges as shown in Table 5. The chaotic DE method provides all costs mostly in last three ranges such as $8^{\text {th }}$ range ( 4 times), $9^{\text {th }}$ range (31 times) and $10^{\text {th }}$ range (65 times). The cost obtained by the chaotic DE method lies in between $120,000 \$ / \mathrm{h}$ to $122,500 \$ / \mathrm{h}$ with 65 times in the $10^{\text {th }}$ range. The proposed GSA, NPSO-LRS and SOHPSO methods provide the all costs obtained in 100 trials in the $9^{\text {th }}$ and $10^{\text {th }}$ ranges only. But, the cost obtained by proposed GSA method lies in between $120,000 \$ / \mathrm{h}$ to $122,500 \$ / \mathrm{h}$ with 92 times in the $10^{\text {th }}$ range as shown in Table 5. Hence, proposed GSA can provide more reliable and quality solutions than MBFA method.

Table 2. 40-unit system with ramp rates and prohibited operating zones

\begin{tabular}{|c|c|c|c|c|c|c|}
\hline Unit & $P i, \min$ & $P i, \max$ & $P_{i 0}$ & $U R_{i}$ & $\overline{D R_{i}}$ & Prohibited Zones $(\mathrm{MW})$ \\
\hline 1 & 36 & 114 & 100 & 114 & 114 & - \\
\hline 2 & 36 & 114 & 100 & 114 & 114 & - \\
\hline 3 & 60 & 120 & 90 & 120 & 120 & - \\
\hline 4 & 80 & 190 & 150 & 100 & 150 & - \\
\hline 5 & 47 & 97 & 80 & 97 & 97 & - \\
\hline 6 & 68 & 140 & 120 & 80 & 125 & - \\
\hline 7 & 110 & 300 & 280 & 165 & 200 & - \\
\hline 8 & 135 & 300 & 200 & 165 & 200 & - \\
\hline 9 & 135 & 300 & 230 & 165 & 200 & - \\
\hline 10 & 130 & 300 & 240 & 155 & 190 & {$[130-150][200230][270-299]$} \\
\hline 11 & 94 & 375 & 210 & 150 & 185 & {$[100-140][230-280][300-350]$} \\
\hline 12 & 94 & 375 & 210 & 150 & 185 & {$[100-140][230-280][300-350]$} \\
\hline 13 & 125 & 500 & 230 & 206 & 235 & {$[150-200][250-300][400-450]$} \\
\hline 14 & 125 & 500 & 355 & 260 & 290 & {$[200-250][300-350][450-490]$} \\
\hline 15 & 125 & 500 & 350 & 186 & 215 & - \\
\hline 16 & 125 & 500 & 350 & 186 & 215 & - \\
\hline 17 & 220 & 500 & 460 & 240 & 270 & - \\
\hline 18 & 220 & 500 & 470 & 240 & 268 & - \\
\hline 19 & 242 & 550 & 500 & 290 & 315 & - \\
\hline 20 & 242 & 550 & 500 & 290 & 315 & - \\
\hline 21 & 254 & 550 & 510 & 335 & 360 & - \\
\hline 22 & 254 & 550 & 520 & 335 & 360 & - \\
\hline 23 & 254 & 550 & 520 & 335 & 362 & - \\
\hline 24 & 254 & 550 & 450 & 350 & 378 & - \\
\hline 25 & 254 & 550 & 400 & 350 & 380 & - \\
\hline 26 & 254 & 550 & 520 & 350 & 380 & - \\
\hline 27 & 10 & 150 & 20 & 95 & 145 & - \\
\hline 28 & 10 & 150 & 20 & 95 & 145 & - \\
\hline 29 & 10 & 150 & 25 & 98 & 145 & - \\
\hline 30 & 47 & 97 & 90 & 97 & 97 & - \\
\hline 31 & 60 & 190 & 170 & 90 & 145 & - \\
\hline 32 & 60 & 190 & 150 & 90 & 145 & - \\
\hline 33 & 60 & 190 & 190 & 90 & 145 & - \\
\hline 34 & 90 & 200 & 190 & 105 & 150 & - \\
\hline 35 & 90 & 200 & 150 & 105 & 150 & - \\
\hline 36 & 90 & 200 & 180 & 105 & 150 & - \\
\hline 37 & 25 & 110 & 60 & 110 & 110 & - \\
\hline 38 & 25 & 110 & 40 & 110 & 110 & - \\
\hline 39 & 25 & 110 & 50 & 110 & 110 & - \\
\hline 40 & 242 & 550 & 512 & 290 & 315 & - \\
\hline
\end{tabular}

Table 3. Comparison of Simulation results between GSA and other methods for 40-unit system

\begin{tabular}{ccccccccc} 
Method & $\begin{array}{c}\text { IFEP } \\
{[15]}\end{array}$ & $\begin{array}{c}\text { MPSO } \\
{[15]}\end{array}$ & $\begin{array}{c}\text { PSO- } \\
\text { LRS[15] }\end{array}$ & $\begin{array}{c}\text { NPSO } \\
{[15]}\end{array}$ & $\begin{array}{c}\text { NPSO- } \\
\text { LRS[15] }\end{array}$ & $\begin{array}{c}\text { SOHPSO } \\
{[14]}\end{array}$ & $\begin{array}{c}\text { Chaotic- } \\
\text { DE [16] }\end{array}$ & GSA \\
\hline $\begin{array}{c}\text { Minimu } \\
\text { m Cost }\end{array}$ & $122,624.3$ & $122,252.2$ & $122,035.7$ & $121,704.7$ & $121,664.4$ & $121,501.1$ & $121,741.9$ & $\mathbf{1 2 1 , 4 4 7 . 5}$ \\
$(\$ / h)$ & 5 & 6 & 9 & 4 & 3 & 4 & 8 & $\mathbf{5}$
\end{tabular}


Table 4. Generation outputs of each generator and the corresponding cost in 40-unit System

\begin{tabular}{|c|c|c|c|c|}
\hline Unit & Pmin & $P \max$ & Gen. (MW) & $\operatorname{Cost}(\$ / h)$ \\
\hline 1 & 36 & 114 & 114.0000 & 978.156 \\
\hline 2 & 36 & 114 & 114.0000 & 978.156 \\
\hline 3 & 60 & 120 & 97.3995 & 1190.547 \\
\hline 4 & 80 & 190 & 179.7330 & 2143.550 \\
\hline 5 & 47 & 97 & 87.7999 & 706.500 \\
\hline 6 & 68 & 140 & 139.9996 & 1596.463 \\
\hline 7 & 110 & 300 & 259.5997 & 2612.885 \\
\hline 8 & 135 & 300 & 284.5996 & 2779.837 \\
\hline 9 & 135 & 300 & 284.5996 & 2798.230 \\
\hline 10 & 130 & 300 & 130.0000 & 2502.065 \\
\hline 11 & 94 & 375 & 167.2422 & 2949.744 \\
\hline 12 & 94 & 375 & 167.2553 & 2967.697 \\
\hline 13 & 125 & 500 & 214.7590 & 3792.067 \\
\hline 14 & 125 & 500 & 394.2754 & 6414.843 \\
\hline 15 & 125 & 500 & 304.5195 & 5171.198 \\
\hline 16 & 125 & 500 & 394.2711 & 6436.551 \\
\hline 17 & 220 & 500 & 489.2793 & 5296.711 \\
\hline 18 & 220 & 500 & 489.2793 & 5288.765 \\
\hline 19 & 242 & 550 & 511.2793 & 5540.929 \\
\hline 20 & 242 & 550 & 511.2794 & 5540.910 \\
\hline 21 & 254 & 550 & 523.2793 & 5071.290 \\
\hline 22 & 254 & 550 & 523.2790 & 5071.290 \\
\hline 23 & 254 & 550 & 523.2794 & 5057.224 \\
\hline 24 & 254 & 550 & 523.2793 & 5057.223 \\
\hline 25 & 254 & 550 & 523.2794 & 5275.089 \\
\hline 26 & 254 & 550 & 523.2793 & 5275.089 \\
\hline 27 & 10 & 150 & 10.0000 & 1140.524 \\
\hline 28 & 10 & 150 & 10.0000 & 1140.524 \\
\hline 29 & 10 & 150 & 10.0000 & 1140.524 \\
\hline 30 & 47 & 97 & 89.4748 & 734.279 \\
\hline 31 & 60 & 190 & 190.0000 & 1643.991 \\
\hline 32 & 60 & 190 & 190.0000 & 1643.991 \\
\hline 33 & 60 & 190 & 190.0000 & 1643.991 \\
\hline 34 & 90 & 200 & 164.7998 & 1585.544 \\
\hline 35 & 90 & 200 & 164.7997 & 1539.870 \\
\hline 36 & 90 & 200 & 164.7998 & 1539.870 \\
\hline 37 & 25 & 110 & 110.0000 & 1220.166 \\
\hline 38 & 25 & 110 & 110.0000 & 1220.166 \\
\hline 39 & 25 & 110 & 110.0000 & 1220.166 \\
\hline 40 & 242 & 550 & 511.2793 & 5540.929 \\
\hline \multicolumn{3}{|c|}{ Total Gen. and Total Cost } & $10,500.000$ & $1,21,447.547$ \\
\hline
\end{tabular}

Table 5. Comparison of different methods on relative frequency of convergence in the ranges of cost $(\mathrm{k} \$ / \mathrm{h})$ for 40-unit System

\begin{tabular}{|c|c|c|c|c|c|c|c|c|c|c|}
\hline $\begin{array}{l}\frac{n}{0} \\
\frac{0}{0} \\
\sum^{0}\end{array}$ & 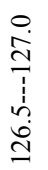 & 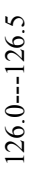 & 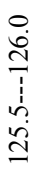 & 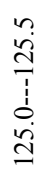 & 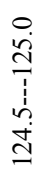 & 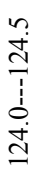 & 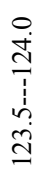 & 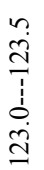 & 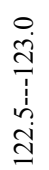 & 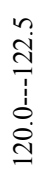 \\
\hline IFEP [15] & 10 & 4 & - & 16 & 22 & 42 & 4 & 2 & - & - \\
\hline MPSO [15] & 6 & - & 4 & 2 & 10 & 20 & 26 & 24 & 6 & - \\
\hline PSO-LRS [15] & - & - & - & - & - & 14 & 26 & 50 & 10 & - \\
\hline NPSO [15] & - & - & 2 & - & 4 & 4 & 18 & 50 & 22 & - \\
\hline NPSO-LRS [15] & - & - & - & - & - & - & - & - & 53 & 47 \\
\hline SOHPSO [14] & - & - & - & - & - & - & - & - & 18 & 82 \\
\hline Chaotic DE [16] & - & - & - & - & - & - & - & 04 & 31 & 65 \\
\hline GSA & - & - & - & - & - & - & - & - & 08 & 92 \\
\hline
\end{tabular}




\section{CONCLUSION}

This paper presents a new stochastic search technique named GSA to solve the non-smooth ELD problem with valve point effect, ramp rate limits constraints and prohibited operating zones constraints. A practical sized ELD test system has been considered. The simulation results demonstrate the effectiveness and robustness of the proposed GSA method to solve ELD problem in modern power systems. The obtained results of the proposed GSA method have been compared with the results obtained from published methods in the literature. The comparison confirms the effectiveness, high quality solution, stable convergence characteristic, good computation efficiency. Hence, the superiority of the proposed GSA method over other heuristic techniques in terms of solution quality is validated. The proposed methodology can be applied to large-scale ELD problems as well optimal dispatch problems under deregulated environment efficiently.

\section{ACKNOWLEDGEMENTS}

The support from the Electrical Engineering Department of Veer Surendra Sai University of Technology, Burla, India, extended to the second author for the work reported in this paper is gratefully acknowledged.

\section{REFERENCES}

[1] A. J. Wood and B. F. Wollenberg, "Power Generation Operation and Control," New York: John Willey and sons, pp. 23-621, 1984.

[2] E. Lin, and G. L. Viviani, "Hierarchical economic dispatch for piecewise quadratic cost functions," IEEE Trans. Power App. Syst., vol. PAS-103, no. 6, pp. 1170-1175, 1984.

[3] D. C. Walters and G.B Sheble, "Genetic Algorithm - economic dispatch example," IEEE Trans. Power Syst., vol. 8, pp. 1325-1332, 1993.

[4] Y. M. Park, J. R. Won, and J. B. Park, "A new approach to economic load dispatch based on improved evolutionary programming," Eng. Intell. Syst. Elect. Eng. Commun., vol. 6, no. 2, pp. 103-110, 1998.

[5] K. P. Wong and Y. W. Wong, "Thermal generator scheduling using hybrid genetic simulated annealing," Proceedings of IEE Transactions on Generation, Transmission and Distribution, vol. 142, pp. 372-380, 1995.

[6] W. M. Lin, F. S. Cheng, and M. T. Tsay, "An improved Tabu search for economic dispatch with multiple minima," IEEE Trans. Power Syst., vol. 17, pp. 108-112, 2002.

[7] Z. L. Gaing, "Particle swarm optimization to solving the economic dispatch considering the generator constraints," IEEE Trans. Power Syst., vol. 18, No. 3, pp. 1187-1195, 2003.

[8] H. Shahinzadeh, S. M. Nasr-Azadani, and N. Jannesari, "Application of Particle Swarm Optimization Algorithm to solving the Economic Load Dispatch of Units in Power Systems with Valve Point Effects", International Journal of Electrical and Computer Engineering, vol. 4, no. 6, pp. 858-867, 2014.

[9] J. H. Park, Y. S. Kim, I. K. Eom, and K. Y. Lee, "Economic load dispatch for piecewise quadratic cost function using Hopfield neural network,” IEEE Trans. Power Syst., vol. 8, pp. 1030-1038, 1993.

[10] K. Y. Lee, A. Sode-Yome, and J. H. Park, "Adaptive Hopfield neural network for economic load dispatch," IEEE Trans. Power Syst., vol. 13, pp. 519-526, 1998.

[11] N. M. Shadaksharappa, "Optimum Generation Scheduling for Thermal Power Plants using Artificial Neural Network", International Journal of Electrical and Computer Engineering, vol. 1, no. 2, pp. 134-139, 2011.

[12] S. O. Orero and M. R. Irving, "Economic dispatch of generators with prohibited operating zones: A genetic algorithm approach," Proc. Inst. Elect. Eng., Gen., Transm., Distrib., vol. 143, no.6, pp. 529-534, 1996.

[13] P. H. Chen and H. C. Chang, "Large-scale economic dispatch by genetic algorithm,"IEEE Trans. Power Syst., vol. 10, no. 4, pp. 1919-1926, 1995.

[14] T. Jayabarathi, G. Sadasivam, and V. Ramachandran, "Evolutionary programming based economic dispatch of generators with prohibited operating zones," Electric Power Systems Research, vol. 52, pp. 261-266, 1998.

[15] A. Pereira-Neto, C. Unsihuay, and O. R. Saavedra, "Efficient evolutionary strategy optimization procedure to solve the nonconvex economic dispatch problem with generator constraints," Proc. Inst. Elect. Eng., Gen., Transm., Distrib., vol. 152, no. 5, pp. 653-660, 2005.

[16] K. T. Chaturvedi, M. Pandit, and L. Srivastava, "Self-organizing hierarchical particle swarm optimization for nonconvex economic dispatch," IEEE Trans. Power Syst., vol. 23, no. 3, pp. 1079-1087, 2008.

[17] A. I. Selvakumar and K. Thanushkodi, "A new particle swarm optimization solution to nonconvex economic dispatch problems," IEEE Trans. Power Syst., vol. 22, No. 1, pp. 42-51, 2007.

[18] L. D. S. Coelho and V. C. Mariani, "Combining of chaotic differential evolution and quadratic programming for economic dispatch optimization with valve point effect," IEEE Trans. Power Syst., vol. 21, no. 2, pp. 989-996, 2006.

[19] E. Rashedi, H. Nezamabadi-pour, and S. Saryazdi, "GSA: A gravitational search algorithm," Information Sciences, vol. 179, pp. 2232-2248, 2009.

[20] S. Duman, U. Guvenc, and N. Yorukeren, "Gravitational search algorithm for economic dispatch with valve-point effects," International Review of Electrical Engineering, vol. 5, no. gff6, pp. 2890-2895, 2010. 
[21] R. K. Swain, N. C. Sahu, and P. K. Hota, "Gravitational search algorithm for optimal economic dispatch," Proceedings of the $2^{\text {nd }}$ International Conference on Communication, Computing \& Security (ICCCS-2012), Procedia Technology, Elsevier, NIT, Rourkela, India, pp.1-6, 2012.

[22] A. K. Barisal, N. C. Sahu, and P. K. Hota, "Short-term hydrothermal scheduling using gravitational search algorithm," Proceedings of the IEEE $2^{\text {nd }}$ International Conference on Power, Control and Embedded Systems (ICPCES-2012), NIT, Allahabad, India, pp.1-6, 2012.

\section{BIOGRAPHIES OF AUTHORS}

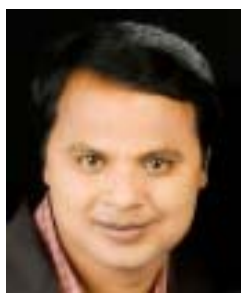

Prakash Kumar Hota was born in 1963 in India. He has graduated in Electrical and Electronics Engineering from the National Institute of Technology (NIT), Tiruchirapalli, India in 1985, received his M.Tech in Industrial Power Control \& Electric Drives in 1992 from Sambalpur University, India and $\mathrm{Ph} . \mathrm{D}$ in Electrical Engineering from Jadavpur University, Kolkata, India in 1999. Currently, he is a Professor of Electrical Engineering and Dean of Sponsored Research and Industrial Consultancy(SRIC) \& Continuing Education Programme (CEP) at Veer Surendra Sai University of Technology (VSSUT), Burla, India. His research interests include Economic Emission Load Dispatch, Hydrothermal Scheduling, Wind Power Generation, Power Quality and Soft Computing applications to different Power System Problems in Deregulated Environment.

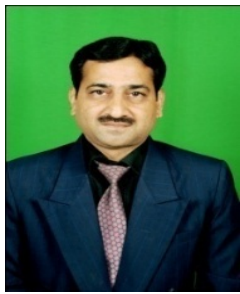

Nakul Charan Sahu was born in Odisha, India in 1965. He received the B.E. degree in Electrical Engineering from Institution of Engineers (India) in 1990 and the M.Tech degree in Power System Engineering from University College of Engineering (Now, VSSUT), Burla, India in 1993. He is a sponsored Ph. D scholar at VSSUT, Burla, India. Currently, he has been with the Electrical Engineering Department, ITER, SOA University, Bhubaneswar, India, where he is an Assistant Professor. His research interests include Economic Operation and Planning of Electrical Power Systems, Artificial Intelligence and Optimization. Currently, he is working as Assistant Professor in Electrical Engineering, ITER, SOA University, Bhubaneswar, India. 